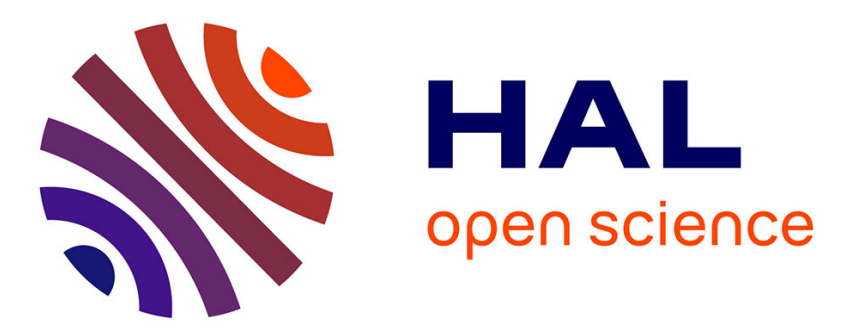

\title{
Autonomic QoS management and supervision system for Home Networks
}

Lionel Molinier, Emna Ghedira Molinier, Mathieu Ligocki, Romain Francois, Guy Pujolle

\section{- To cite this version:}

Lionel Molinier, Emna Ghedira Molinier, Mathieu Ligocki, Romain Francois, Guy Pujolle. Autonomic QoS management and supervision system for Home Networks. WD 2009 - 2nd IFIP Wireless Days Conference, Dec 2009, Paris, France. pp.1-6, 10.1109/WD.2009.5449648 . hal-00631619

\section{HAL Id: hal-00631619 https://hal.science/hal-00631619}

Submitted on 12 Oct 2013

HAL is a multi-disciplinary open access archive for the deposit and dissemination of scientific research documents, whether they are published or not. The documents may come from teaching and research institutions in France or abroad, or from public or private research centers.
L'archive ouverte pluridisciplinaire HAL, est destinée au dépôt et à la diffusion de documents scientifiques de niveau recherche, publiés ou non, émanant des établissements d'enseignement et de recherche français ou étrangers, des laboratoires publics ou privés. 


\title{
Autonomic QoS management and supervision system for Home Networks
}

\author{
Lionel Molinier* ${ }^{*}$, Emna Ghedira*, Mathieu Ligocki ${ }^{\dagger}$, Romain Francois ${ }^{\dagger}$ and Guy Pujolle* \\ *Paris Universitas - LIP6, Paris, France \\ Email: \{lionel.molinier, emna.ghedira, guy.pujolle\}@lip6.fr \\ ${ }^{\dagger}$ Ginkgo Networks, Paris, France \\ Email: \{lionel.molinier, mathieu.ligocki, romain.francois\}@ginkgo-networks.com
}

\begin{abstract}
Broadband access to the Internet at home was the first step in the emergence of so called Home Networks. In a close future, the number of appliance connected will rise and the network will become the home backbone. This lead us to a mesh architecture, in which routing will be mandatory. This paper introduces a complete system to, first, pilot the forwarding in order to ensure a proper quality of experience; and secondly to monitor and diagnosis the network from the ISP-network in order to reduce OPEX. The system is based on the knowledge plane composed of agents embedded on devices.
\end{abstract}

Index Terms-Home Network, WiFi, HomePlug AV, Agent, QoS, Knowledge Plane, Ontology, Throughput estimation, Supervision

\section{INTRODUCTION}

The spread of broadband accesses and of digital TVs at home is boosting the emergence of so called Home Networks. Currently, Home Networks are only composed of a broadband gateway, a set-top box and the domestic PC. We expect in a close future IPTV, VoIP WiFi phones, PDA, mobile phones, sensors and even domestic appliances (refrigerator, security devices) to be connected as well.

Contrary to classical networks, the Home Network is not monitored nor configured by an administrator. The end-user is supposed to be a neophyte, without any network skills. This implies strong constraints and the network needs to implement self-* functionalities, mainly:

- self-configuration: a new device should be easy to connect;

- self-healing: link disruption or device failures must be transparent for the end-user, if possible;

- self-optimizing: the network must reconfigure itself to tackle potential resource shortage.

Those functionalities lead us to autonomic networks techniques, and mainly distributed ones.

In this paper, we first, in section I present a widely accepted Home Network architecture. However, to fit the requirements of self-* capabilities, he have used Distributed Artificial Intelligence as explained in II. In III we are presenting in details our solution and illustrate the testbed implemented IV, which complete the work presented in [1], [2]. The multi-agent system involved in those solutions is mostly the same which permits us, in $\mathrm{V}$, to compare them and also classical ones. Finally, we are concluding this paper by pointing out some work that remain to be achieved in section VI.

\section{HOME NETWORK DEFINITION}

Major initiatives are working on the Home Network for several years now, in order to identify this emerging network, the use-case, the technologies to be involved and so on. The Home Network cannot be treated separately from its broadband access to the Internet.

\section{A. Home Network overview}

The Broadband Forum is one of the oldest workgroup that mainly focuses on the broadband access. However, since the last years, the Home Network evolution has been taken into account in terms of management. Therefore, the TR069 [3] jointly with the TR-098 [4] describe and complete and powerful management architecture that tackles both the Home Gateway and others in-home devices. For instance the TR-104 [5] describe a data model for a VoIP end device. However, the Home Network architecture remains out of the scope of the Broadband Forum.

However, this last point is covered by the Home Gateway Initiative (HGI) which is working on defining a wide set of use cases, and on enumerating technical solutions that could be used in a future Home Network. The purpose of this initiative is to mutualize development effort. The main idea is to enable a user to connect all its devices smoothly. For instance, an user must be able to watch a movie stored on the living room DVR directly on its bedroom TV.

Regarding the application, the DLNA [6] (Digital Living Network Alliance) is focusing on the software part of the upcoming use. Devices, DLNA compliant, are able to communicate and exchange content, like in the previously mentioned use case. It is an extension of the UPnP architecture, where the service discovery and management is implemented. Moreover, it defines how audio or video stream should be transported on the network, for instance in terms of codecs.

\section{B. Architecture}

In nowadays architecture, several independant technologies are running, such as WiFi, DECT ... However, the IP convergence is in progress, and the number of appliances connected will rise pointing out limitation of the star topology. Moreover, the Home Network will have to handle multiple technologies, such as WiFi, HomePlug AV, ZigBee, Ethernet, Bluetooth... 
At the same time, the huge bandwidth increase of the broadband access enable users to enjoy $100 \mathrm{Mb} / \mathrm{s}$ or even more till the gateway. However, the WiFi alone cannot support such bandwidth, with a wide coverage. A simple and usual way to increase the overall bandwidth is to decrease even more cell range.

The foreseen architecture is taking into account those points, as $H N I D^{1}$ are introduced. They have several functionalities, such as realizing a transparent bridge between technologies, and to extend wireless coverage. This defines 2 stages in the network.

The first level is a full-mesh backbone of those bridging devices. They can connect to each other using Ethernet, PLC, MoCA, or any wireless technology. Connection diversity introduces several path to reach any other bridge. A smart routing scheme can be used to ensure a proper QoS. Those devices can be seen as wireless extenders.

The second level is for end devices, that can connect directly to any bridge. The connection can be either wireless, or wired. The figure 1 presents a typical instantiation of the architecture.

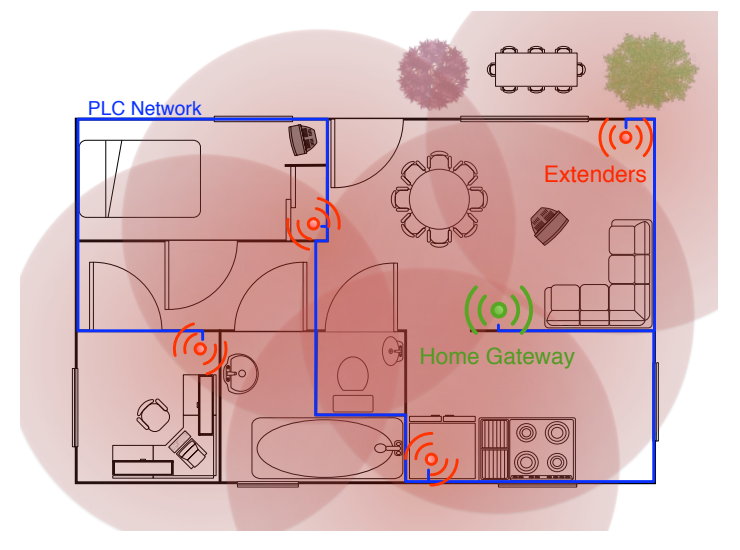

Fig. 1. Home Network architecture

\section{Routing concerns}

In the section I-B we have presented a mesh-hybrid architecture and the shared and perturbation sensitive transmission technology are tricky for the routing scheme.

Providing a routing protocol into such networks is not something difficult. There is a lot of routing protocols in the ad-hoc networks literature. The two mains are AODV [7] or OLSR [8]. Both are providing connectivity but without any QoS concerns. Some QOS extensions has been proposed, and recently the Hybrid Wireless Mesh Protocol (HWMP) has been standardized in the 802.11s. It introduces the Air Metric in order to introduce QoS in the routing protocol. Unfortunately, this protocol is only for WiFi ad-hoc networks, and does not encompass our problematic.

Moreover, in the Home Network we have to ensure a good Quality of Experience for the end user, so the HWMP approach is not sufficient anyway. Indeed, the network should

\footnotetext{
${ }^{1}$ HNID: Home Network Infrastructure Device
}

not only select the best path available, but must spread out the load over different link so that each flow can enjoy the appropriate QoS.

As HWMP, we have adopted a layer 2 approach, based on STP, patched to permit our system to benefit from diversity. The STP is computing the main path and our system is able to change some path to avoid problematic situations, in which WiFi or HPAV links capacity is fading down, due to electromagnetic perturbations.

\section{AgEnt-BASED SOLUTION}

As the architecture is complexe, we focus on autonomic networking, and more precisely on the knowledge plane concept implemented using a multi-agent system.

\section{A. Knowledge plane}

Introduced by [9], the knowledge plane can handle this complexity with a reasonable computation overhead. This plane was defined as

[...] a distributed and decentralized construct within the network that gathers, aggregates, and manages information about network behavior and operation.

The knowledge plane enables the network to implement the self-* capabilities. Those requirements can be satisfied by a multi-agent system, which provides a decentralized approach to solve problems in complex environments [10]. The followings sections are introducing the agent (see II-B). We are finishing with section II-C and II-D in which we are describing the platform developed.

\section{B. Agent overview}

According to J. Ferber in [11], an agent can be a phyisical entity that can perceive and act on its environment. However, in our solution, we are using intelligent agents which can be defined as:

[an intelligent agent can] communicate with others, is autonomous and has skills to achieve its goals and tendencies.

Given this definition, we can exhibit some fundamontal characteristics that must have an agent, as the work done by M. Wooldridge, in [12]. We denote autonomicity, social ability, reactivity and pro-activity. Developped agent are fitting most of those charactiristics.

\section{Ginkgo MAS}

An intelligent agent, as defined in section II-B, is embedded on each network device as shown on figure 3 . As previously explained, the Ginkgo agent, as an environment entity, has a knowledge representation, a perception of its environment which are both stored in a situated view, which is defined in [13].

It is mandatory to have a common vocabulary, both for the inter-agent communication and for the knowledge representation. Therefore an ontology has been developed. The figure 2 is 


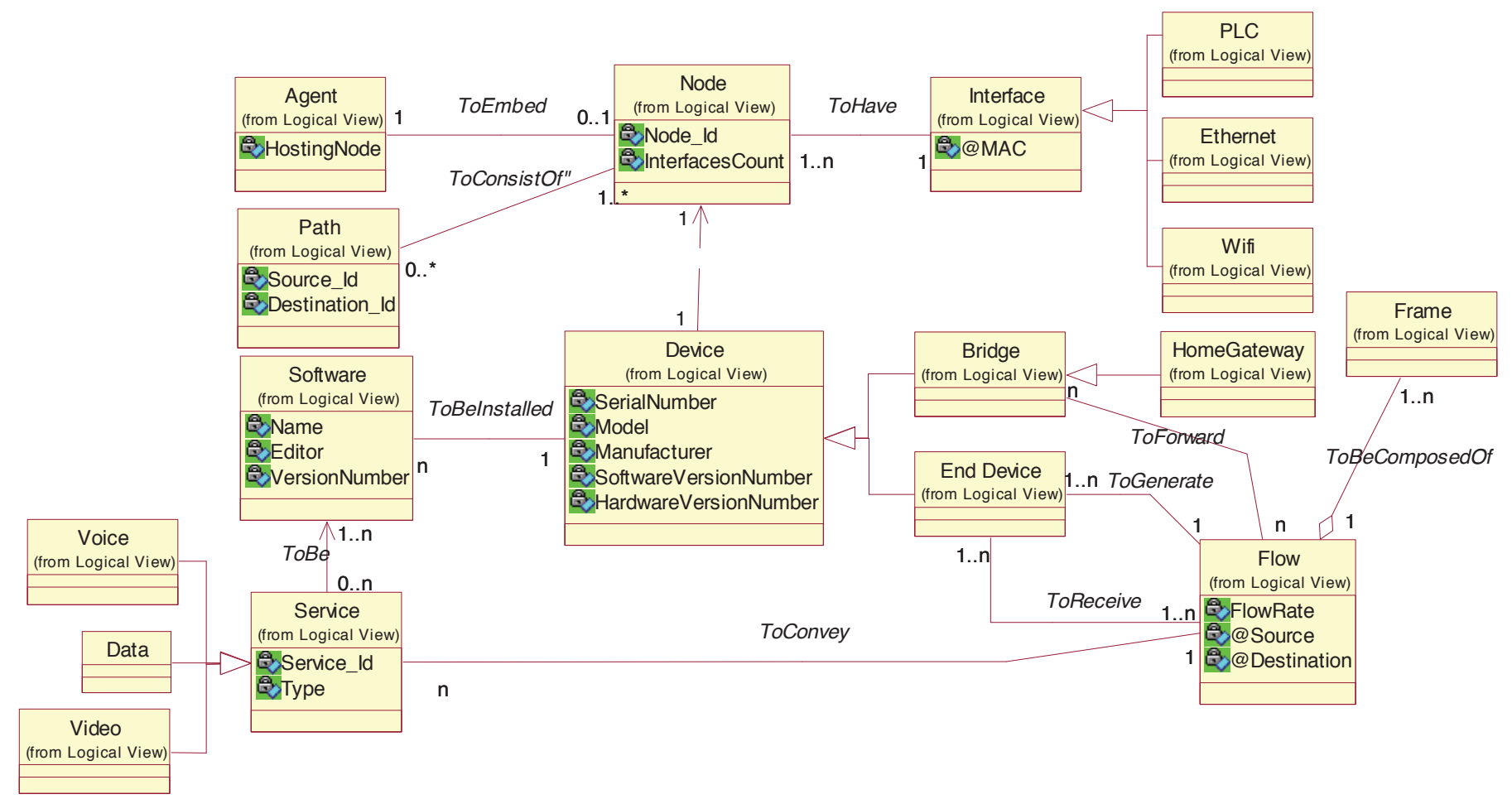

Fig. 2. Extract of the developped ontology

representing a subpart of the ontology using Unified Modeling Language (UML).

The inter-agent communication in the Ginkgo MAS is done via the situated view exchange. In other words, there is no speeching $a c t^{2}$, but only knowledge exchange. In a MAS, each agent has a partial view of its environment, the Ginkgo MAS has been designed so that an agent can only exchange his situated view with its directly connected neighbors (1-hop neighbors), as shown on figure 3 .

\section{Agent description}

As shown on figure 4, agent capabilities are known as behaviors in the Ginkgo agent and are implemented using plug-in module. This set of behaviors is orchestrated by the dynamic planner which can start, stop, configure or even modify behaviors. The agent's knowledge is stored in the situated view which gather both remote and local knowledge. A behavior can sense or act on the hosting node using the effector and sensor interfaces.

\section{Ginkgo agent in the Home Network}

In a Home Network context, agent's goal is to change quickly a path when the available resources does not meet flows requirements anymore. For now we have selected 2 different situations:

\footnotetext{
${ }^{2} \mathrm{~A}$ speech act is a intentional action performed within a communication. There are 5 types of speeching act: representative (it's sunny), directive (Open the window), commisive (I'll assist to the meeting), expressive (I'm happy!) and declarative (The court declares the defendant not guilty)
}

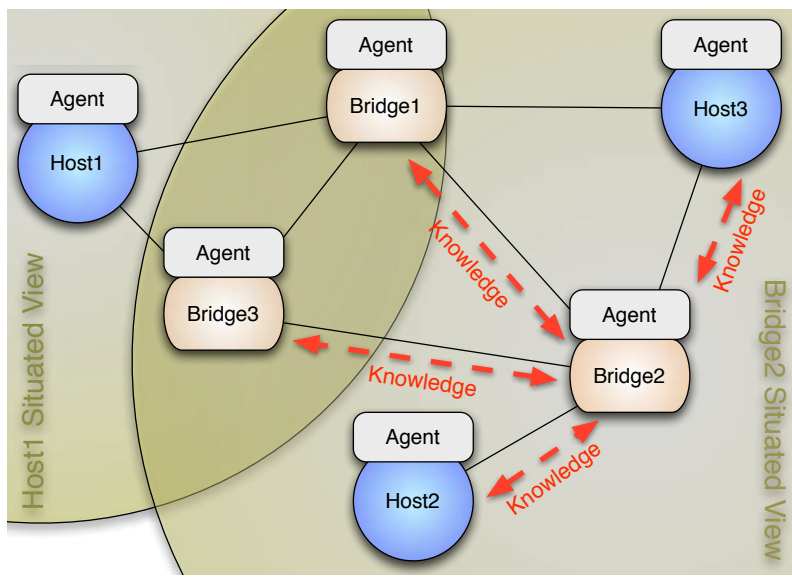

Fig. 3. Agents situated view concept

- link disruption: agents must change the path as soon as a link failure is detected;

- link quality degradation: agents must monitor the link quality, which means the available bandwidth ${ }^{3}$. If a noticeable degradation occurs, agents should change the path.

\footnotetext{
${ }^{3}$ This is an approximation to consider the QoS as a matter of bandwidth, but in most cases, the delay can be converted into extra bandwidth. This works if the delay is considered to be caused only by the serialization problem on the link.
} 


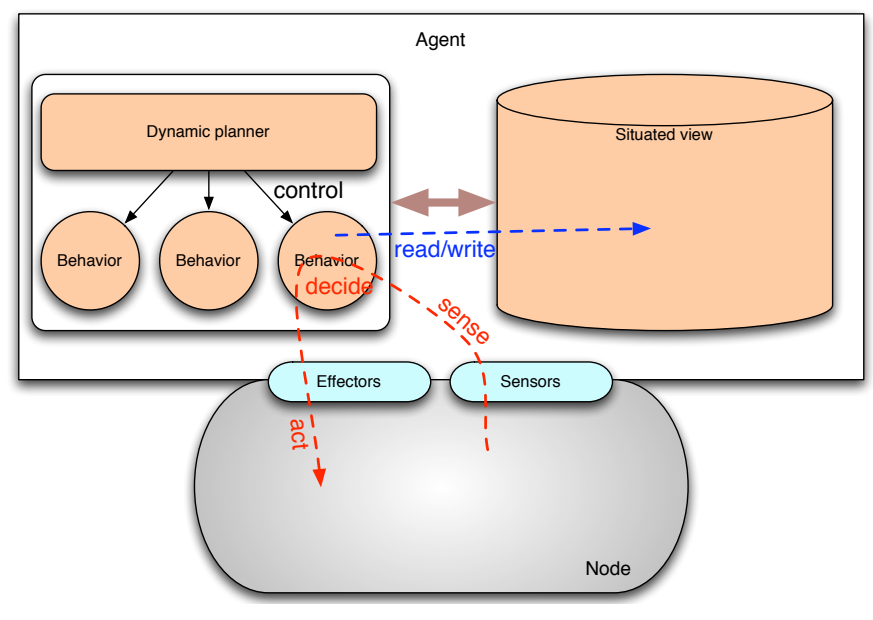

Fig. 4. Agent operation

\section{A. Building alternatives routes}

In order to ensure a proper QoS in the Home Nework, agents are maintaining alternatives routing tables so that they can switch quickly on those alternative if the main route does not suceed in carring flows with the proper QoS. Those alternative are computed proactively, before any problem occurs.

The alternative route computation problem is similar to a task allocation one in a multi-agent system. Indeed, we can consider that finding an alternative route for a given destination, for a given service is a task. One the most used mechanism is a auction process. We have designed a seald first-price auction, which is the auction in the common sense except that biders can do only one bid without knowing other bids.

In more details, when an agent is detecting a new flow transiting though itself, he starts an auction which is then diffused thorugh the situated view. In the meanwhile, when an agent is detecting a new auction in the situated viex he tries to make a bid, regarding its resources. The first agent is waiting, and then selects the best bid available and registers the bidder as alternative next hop.

\section{B. Link monitoring}

Agents have to monitor the link status and performance in realtime, since they have to change as fast as possible the route. These constraints prevent us from using any active measurement methods. Everything must be done locally, in the hosting equipment. Moreover, the computation cost must be taken into account. In a first step of our work, we have elaborated methods as simple as possible 4 to have references for any further works. This section discusses quickly the link status of each interface, and then the maximum available bandwidth estimation on $\mathrm{WiFi}$, and finally on HomePlug AV links.

\footnotetext{
${ }^{4}$ The strongest approximation is the CSMA/CA effect on shared mediums.
}

1) WiFi capacity estimation: The algorithm used to estimate the WiFi bandwidth is at this step of our work very simple. In digital communication on noisy channel, the physical layer has to optimize the tradeoff between transmission bit rate and noise resilience on the channel. Some algorithms exist to approach this optimality, such as Atsushi Onoe [14] algorithm or SampleRate described in [15]. We are using this last one in our experiments ${ }^{5}$.

In order to operate successfully, this algorithm tries each modulation regularly to compute performance metric. In average, it generally uses to best modulation. The tricky point is to test appropriate modulations while not noticeably degrading overall performances. Our capacity estimation is based on those statistics, which are preprocessed before being input of a neural network which outputs the estimation.

The neural networks designed allows us to compute in realtime, with a quite small computation overhead, the available capacity on the link. The figure 5 shows the real value and the estimated one on the same graph.

2) HomePlug $A V$ capacity estimation: As for the $\mathrm{WiFi}$, the HomePlug AV physical layer as to adapt to the channel conditions by adjusting the modulation. However, despite the $\mathrm{WiFi}$, there are 1152 frequencies available for the communication (see [16] for more information). The resulting modulation table is called the Tone Map, from which we can compute the channel capacity, called $c a p_{c}$.

The HomePlug AV chip is also maintaining statistics on successfully transmitted frames ${ }^{6}$ and unsuccessfully ones. We are calling $p_{s u c}$ the proportion of successfully transmitted frames.

Since there is a high binary error rate on power lines, the HomePlug $\mathrm{AV}$ is using a powerful $\mathrm{FEC}^{7}$ system based on Turbo Codes. This adds overhead in blocks, so that the payload bandwidth is reduced by a factor $p_{F E C}$. As explained in [17], the factor value is $p_{F E C}=\frac{1}{2}$.

Finally, the bandwidth can be computed by the formula 1 :

$$
h p a v \_b w_{\max }=p_{s u c} \cdot p_{F E C} \cdot c a p_{c}
$$

In practice, we are smoothing this value so that high frequency variation are filtered out. The figure 5 is showing the performance of our bandwidth estimation.

\section{Home Network supervision}

The agent embedded on each device is aquiring knowledge, so it is simple to process knowledge so that the agent can extract noticeable changes. Those changes generates events, which are stored in each agent knowledge base. A dedicated neighborhood is defined in the situated view, so that each agent has the gateway as neighbor. This virtual neighborhood allows us to propagate event to the gateway. The gateway is then proprosing a web-service API, as shown in figure 4.

\footnotetext{
${ }^{5}$ There is no strict limitation in using SampleRate, since most of the rate selection algorithm are using the same principle.

${ }^{6}$ In HomePlug AV, we prefer talking of blocks

${ }^{7}$ FEC: Forward Error Correction
} 


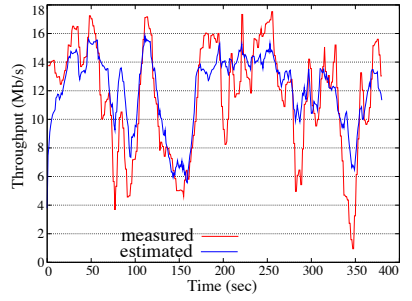

(a) $\mathrm{WiFi}$

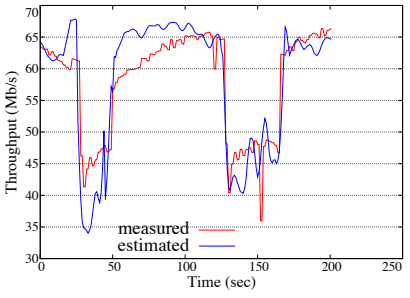

(b) HomePlug AV
Fig. 5. Capacity estimation performance measurements.

Based on this knowledge distribution, any kind of webservice-enabled interface can access to this knowledge. In our system we are it in order the trace the network topology evolution along the network existence. This point is very helpful to diagnosticate problems, and identify the HNID from which it originates. The figure 6 is presenting some possible location of such interfaces, either, in the home, user-oriented, either, in the ISP network technician-oriented.

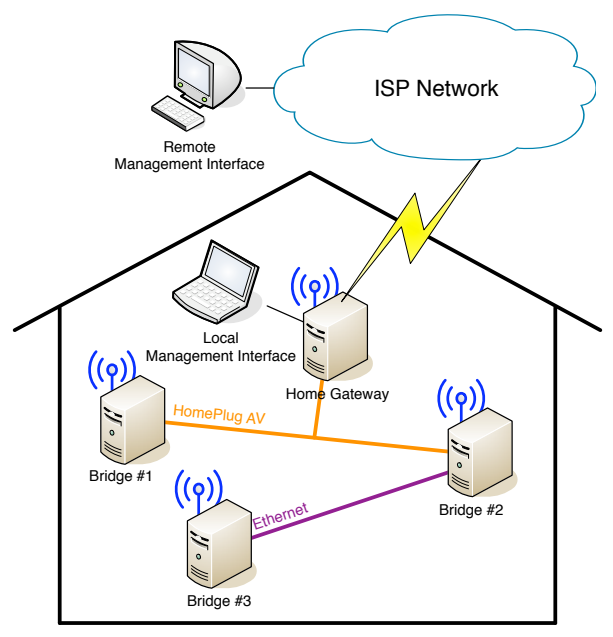

Fig. 6. Supervision architecture

\section{EXPERIMENTAL SET-UP}

To experiment previous concepts, we have decided to setup a real testbed instead of using a simulator because many potential problems can be caused by implementation shortcuts. We have simplified the architecture to only $4 \mathrm{PC}$ running GNU/Linux, in order to isolate problems quickly. The figure 7 is illustrating the testbed. This testbed implement both a basic (yet functionnal) supervision system and a QoS management one. This last one can support congestion scenario, or even perturbation ones, or even link disruption.

The testbed has already been presented in details in previous work, as perturbation scenarios. This paper will focus a the congestion scenario and the supervision implementation, as described in III-C.

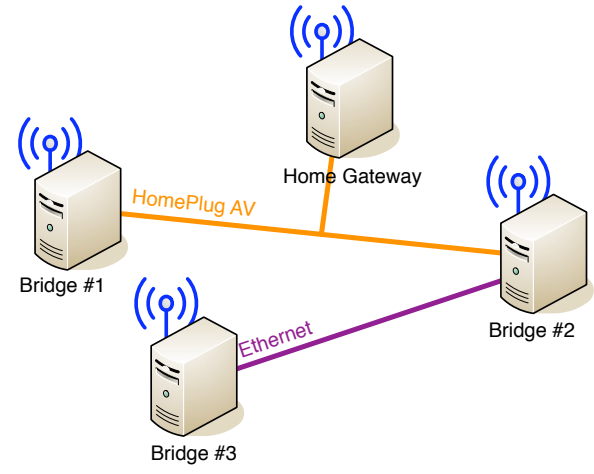

Fig. 7. Testbed network

\section{Results}

Given the network architecture presented in IV, we have realized two sets of experimentations which complets the one done in [2]. The first one was performed to check agents ability on detecting congestion on a link. The second was to check agents functionnality in their supervision task, such as history recording for instance.

\section{A. Dealing with congestions}

The system is able to monitor and to prevent from congestion over a given link. In this use-case, we are generating 2 flows, one video flow and one data flow. The video flow has to be protected from any congestion ${ }^{8}$. The figure 8 is illustrating the steps of the scenario.

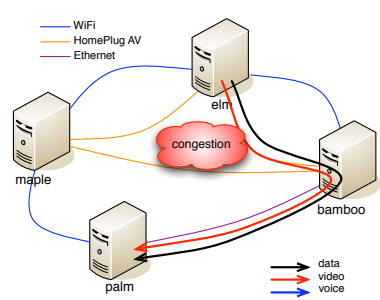

(a) Before

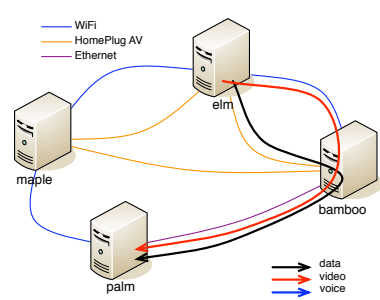

(b) After
Fig. 8. Congestion scenario

We have subjectively meseaured that the recovery time is under the half second. A complete measurement system has not been implemented yet. However, this is unperceivable on the video.

\section{B. Supervision}

We have developped a GUI to test our agent in the supervision function. Given the current topology and a list of noticable events that had occur in the network, this interface is able to bactrace topology change, which mean that it can display the topolgy at any time.

\footnotetext{
${ }^{8}$ This is a goal given to the agent, via the dynamic planner
} 


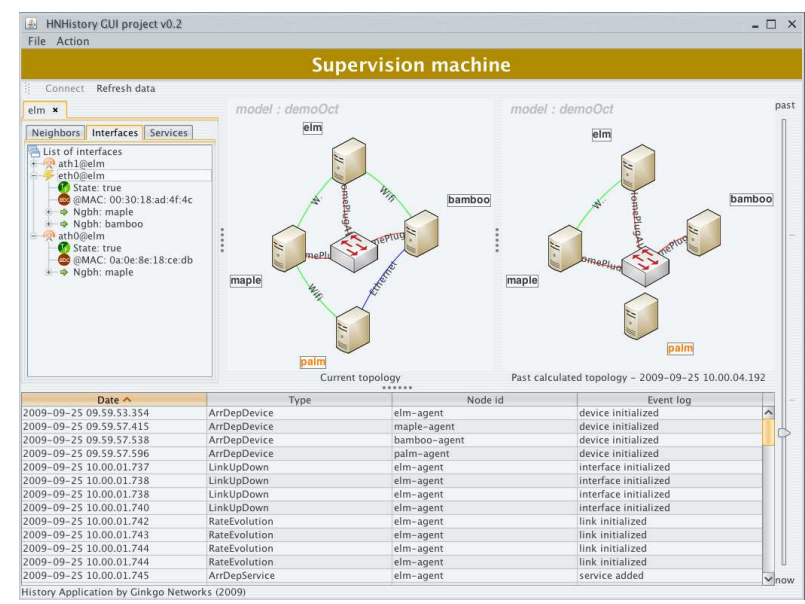

Fig. 9. Screenshot of supervision interface

\section{FUTURE WORKS}

In previous sections, we have presented the fourth step of our work. The first one was to simulate the knowledge plane on an hand-made simulator to validate agents operations. We have setup, as a second step, a real testbed using a layer 3 routing protocol. Then we switch to the layer 2 approach of routing, and finally we have refined our system and added the supervision part. However a lot of work remains to be done, mainly regarding devices, extensive measurements and validation.

The architecture used for these experimentations is not yet complete, and one or two more PC will be added soon. This will enable more complex scenario, where agents will have to cooperate more intensively.

The passive QoS measurements both for WiFi or HomePlug AV is still under study to improve the confidence in the estimator in one hand, and in the other hand to handle the CSMA/CA effect. At the same time the agent framework is used to simulate the CSMA/CA behavior. Moreover a statistical validation of the work is in progress to ensure that the routing is consistent in any situation.

For the WiFi, the WDS allows horizontal handovers but it has been eluded. Next work will be to integrate an algorithm to optimize the handover decision based on several criteria, such as the number of stations connected, the required bandwidth... The paper [18] presents the handover work on the same SMA platform that can be integrated with this work.

\section{CONCLUSION}

Home Networks are using perturbation sensitive technologies due to their low deployment costs. However, users are requiring high quality of service as this network is the backbone of tomorrow homes. Dealing with this antagonism is not as simple as elaborating a new protocol. It as been experimented in $\mathrm{V}$ that a multi-agent system is able to master such complex environments.

Futhermore, if the number of device increase, the complexity is rising which may lead to unacceptable OPEX for the ISP.
An efficient supervision system has to designed at the same time. Our first work are already conclusive and points out that an autonomic solution based on a MAS is able to perform in those networks.

Our first real experimentations are quite satisfying as they can solve partially many problems. The real field is still far, but we believe that this work is nice first step, so that we will work to reach real Home Network devices.

\section{ACKNOWLEDGMENT}

The authors would like to thank the Ginkgo Networks developer team that helps them in implementing this solution, and one of our closest CELTIC/AutHoNe partner, Orange Labs for their guidelines and expertise on such networks.

\section{REFERENCES}

[1] G. Nguengang, L. Molinier, J. Boite, D. Gaïti, and G. Pujolle, "Intelligent routing scheme in home networks," in Home Networking, Springer, Ed., 2008, pp. 179-196.

[2] L. Molinier, M. Ligocki, G. Pujolle, and D. Gaiti, "Piloting the spanning tree protocol in home networks using a multi-agent system," Nov. 2008, pp. $1-5$.

[3] W. Lupton, J. Blackford, M. Digdon, and T. Spets, "Cpe wan management protocol - amendment 2," Broadband Forum, Tech. Rep., 2007. [Online]. Available: http://www.broadband-forum.org/technical/ download/TR-069Amendment2.pdf

[4] W. Lupton and C. Bouchat, "Internet gateway device data model for tr-069 - amendment 2," Broadband Forum, Tech. Rep., 2008. [Online] Available: http://www.broadband-forum.org/technical/download/TR-98_ Amendment_2.pdf

[5] J. Bernstein and B. Stark, "Dslhome provisioning parameters for voip cpe," Broadband Forum, Tech. Rep., 2005.

[6] "Dlna overview and vision whitepaper," Digital Living Network Alliance, Tech. Rep., 2007. [Online]. Available: http://www.dlna.org

[7] C. Perkins, E. Belding-Royer, and S. Das, "Ad hoc on-demand distance vector (aodv) routing," RFC 3561 (Experimental), jul 2003. [Online]. Available: http://www.ietf.org/rfc/rfc3561.txt

[8] T. Clausen and P. Jacquet, "Optimized link state routing protocol (olsr)," RFC 3626 (Experimental), oct 2003. [Online]. Available: http://www.ietf.org/rfc/rfc3626.txt

[9] D. D. Clark, C. Partridge, C. J. Ramming, and J. T. Wroclawski, "A knowledge plane for the internet," in SIGCOMM '03: Proceedings of the 2003 conference on Applications, technologies, architectures, and protocols for computer communications. New York, NY, USA: ACM Press, 2003, pp. 3-10. [Online]. Available: http: //portal.acm.org/citation.cfm?id=863957

[10] M. Wooldridge, An Introduction to Multi-Agent Systems. New York, NY, USA: John Wiley \& Sons, Inc., 2001.

[11] J. Ferber, Multi-Agent Systems: An Introduction to Distributed Artificial Intelligence. Addison-Wesley Professional, February 1999.

[12] M. Wooldridge and N. R. Jennings, "Intelligent agents: Theory and practice," Knowledge Engineering Review, vol. 10, no. 2, pp. 115-152, 1995. [Online]. Available: http://citeseerx.ist.psu.edu/viewdoc/ summary?doi=10.1.1.55.2702

[13] T. Bullot, R. Khatoun, L. Hugues, D. Gaïti, and L. Merghem-Boulahia, "A situatedness-based knowledge plane for autonomic networking," Int. J. Netw. Manag., vol. 18, no. 2, pp. 171-193, 2008.

[14] [Online]. Available: http://madwifi.org

[15] J. Bicket, "Bit-rate selection in wireless networks," Ph.D. dissertation, Massachusetts Institute of Technology, 2005.

[16] M. E. Hazen, "The technology behind homeplug av powerline communications," Computer, vol. 41, no. 6, pp. 90-92, 2008.

[17] L. Guerrieri, P. Bisaglia, G. Dell'Amico, and E. Guerrini, "Performance of the turbo coded homeplug av system over power-line channels," March 2007, pp. 138-143.

[18] M. Abid, M. Ligocki, L. Molinier, G. Nguenguang, G. Pujolle, D. Gaiti, and H. Zimmermann, "Practical handover optimization solution using autonomic agent-based piloting system," Nov. 2008, pp. 1-5. 